\title{
Hybrid PAPR Reduction Technique for Complex Wavelet Packet Modulation System
}

\author{
Hikmat N. Abdullah, Fadhil Sahib \\ Electrical engineering department \\ Al-Mustansiryah university \\ Baghdad, Iraq
}

\author{
Alejandro Valenzuela \\ EMT department \\ H-BRS university of applied sciences \\ Bonn, Germany
}

\begin{abstract}
High peak to average power ratio (PAPR) of a transmitted signal is one of the major drawbacks of the complex wavelet packet modulation (CWPM) as usual in any multicarrier communication system. Utilizing the advantage of concentrating the energy to certain subspaces of the discrete wavelet transform, many PAPR reduction techniques are proposed to solve this problem like threshold and clipping methods. In this paper a novel hybrid PAPR reduction method for CWPM called Threshold-Clipping (TC) method has been proposed. The simulation results in Rayleigh multipath fading channel show that the proposed scheme has achieved $4.5 \mathrm{~dB}$ and $3 \mathrm{~dB}$ reduction in PAPR over the traditional threshold and clipping methods respectively with less than $0.5 \mathrm{~dB}$ degradation in bit error probability.
\end{abstract}

Keywords-component; Peak to average power ratio; complex wavelet transform; complex WPM; multicarrier modulation;

\section{INTRODUCTION}

Wavelet Packet Modulation (WPM) is a multiplexing method where information carrying bits modulate a set of orthogonal wavelet packet waveforms which are then combined into a single composite signal. It is a promising alternative to the popular Fast Fourier Transform (FFT) based Orthogonal Frequency Division Multiplexing (OFDM) [1]. However, high Peak to Average Power Ratio (PAPR) that affects OFDM is also a problem in WPM. The large peaks increase the amount of inter-modulation distortion resulting in an increase in the error rate. The average signal power must therefore be kept low to ensure that the transmitter amplifier operates in the linear region. Many proposals to has been presented to reduce the PAPR in WPM systems [2-6].

Complex Wavelet Packet Transform (CWPT) is an elegant computational structure introduced in [7]. It is a form of discrete wavelet transform, which generates complex coefficients by using a dual tree of wavelet filters to obtain their real and imaginary parts. The CWPT transform have the following features: good directional analysis, low redundancy, improved shift-invariance property, simplicity of implementation and reduced computational cost. In [8] Complex Wavelet Packet Modulation (CWPM), that uses CWPT in principle, is proposed as an efficient multicarrier modulation scheme. It has been shown that CWPM have better BER performance than WPM and most multicarrier modulation schemes based on OFDM. However, although the PAPR reduction techniques proposed for WPM are applicable for CWPM, the performance evaluation of using these schemes in CWPM is not well studied.

In this paper the performance of three PAPR reduction techniques for CWPM system is evaluated in noisy and Rayleigh multipath fading channels. These are: threshold method, clipping method and a proposed hybrid technique composed of both the threshold and clipping methods.

\section{COMPleX WaVelet Packet Modulation System}

Fig.1 illustrates a generic block diagram of CWPM system. It employs two filter banks: Inverse Discrete Wavelet Packet Transform (IDWPT) (reconstruction) placed at the transmitter side, and Discrete Wavelet Packet Transform (DWPT) (decomposition) placed at the receiver side. The block "MAKE CMPLX" accepts two N-dimensional real vectors as inputs. Its output is an $\mathrm{N}$-dimensional complex vector whose ith complex element is formed from the ith real elements of the two input vectors. The input to the inverse wavelet transform is DQPSK complex symbols, therefore, there are two IDWPT blocks: one for real symbols and the other for imaginary ones. The output of the two IDWPTs is combined together in complex form to produce the transmitted signal $\mathrm{x}[\mathrm{n}]$. The transmitted signal is constructed as the sum of $\mathrm{N}$ waveforms $\varphi_{\mathrm{j}}[\mathrm{k}]$ (wavelet packets) individually modulated with the DQPSK symbols as follows:

$$
x[n]=\sum_{k}\left(\sum_{j=0}^{N-1} a_{k, j} \varphi_{j}(n-k N)+i \sum_{j=0}^{N-1} b_{k, j} \varphi_{j}(n-k N)\right)
$$

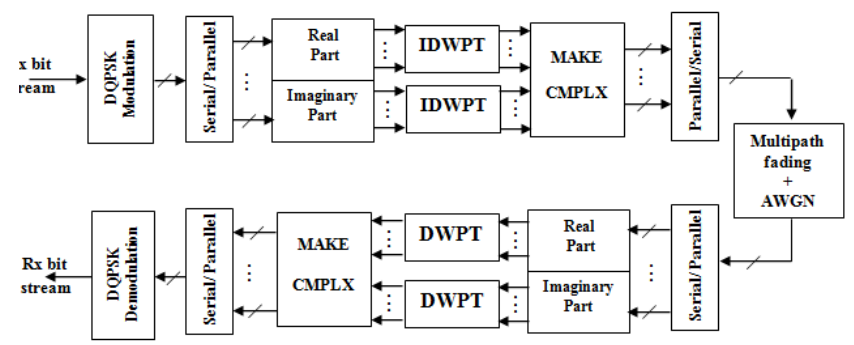

Figure 1. Block diagram of the CWPM system. Arrows with a strike indicate complex quantities[8]. 
where $a_{k, j}, b_{k, j}$ are the real and imaginary constellations encoded kth data symbol modulating the jth wavelet packet basis function respectively. The DWPT at the receiver recovers the transmitted symbols $a_{k, j}$, $b_{k, j}$ through the analysis formula exploiting orthogonality properties of DWPT.

\section{Peak to Average Power Ratio Reduction}

The PAPR of the base band transmitted signal $x(t)$ is defined as the ratio of maximum power of the transmitted signal over the average power. The PAPR of discrete time signal is given as [6]:

$$
\text { PAPR }=\frac{\max _{n}\left(|x(n)|^{2}\right)}{E\left(|x(n)|^{2}\right)}
$$

where $E($.$) denotes ensemble average calculated over the$ duration of WPDM symbols. The Complementary Cumulative Distribution Function (CCDF) of the PAPR is one of the most frequently used performance measures for PAPR reduction techniques. It denotes the probability that the PAPR of data block exceeds a given certain value PAPR0, and is expressed as follows [2]:

$$
\begin{aligned}
\operatorname{CCDF}(\mathrm{PAPR} 0) & =\operatorname{Pr}\{\mathrm{PAPR}>\mathrm{PAPR} 0\} \\
& =1-\left(1-e^{-P A P R 0}\right)^{N}
\end{aligned}
$$

where $\mathrm{N}$ is the number of subcarriers.

\section{A. The threshold method}

The CDWT analyses a finite length time domain signal by breaking up the initial domain in two parts: the details and the approximated information. The approximation domain is successively decomposed into detail and approximation domains and so on. Since the DWT is scattered over many domains, only few coefficients of DWT dominates the representation. This can reduce the PAPR with little reconstruction loss [3]. Let $\mathrm{x}(\mathrm{n})$ be the signal obtained after orthogonal modulation. Since wavelet transforms always concentrate energy on some given number of bases, we can introduce a threshold $\mathrm{T}$ and compare it with the energy of each orthogonal base. The thresholding function is given as [5]:

$$
x_{T}(n)= \begin{cases}0 & \text { if }|x(n)|<T \\ x(n) & \text { if }|x(n)| \geq T\end{cases}
$$

where $\mathrm{x}_{\mathrm{T}}(\mathrm{n})$ represents the output value after thresholding and $\mathrm{T}$ is threshold value. Donoho et al. [9] introduced various shrinkage rules based on different threshold values and thresholding functions such as "visushrink" with fixed universal threshold:

$$
\mathrm{T}=\sigma \sqrt{2 \log \mathrm{N}}
$$

with

$$
\sigma=\frac{\operatorname{MAD}(\mathrm{x})}{0.6745}
$$

where $\operatorname{MAD}(\mathrm{x})$ is median absolute deviation of $\mathrm{x}(\mathrm{n})$.

\section{B. The clipping method}

The clipped multicarrier signal is presented as [10]:

$$
x_{c}(n)= \begin{cases}x(n) & \text { if }|x(n)| \leq A_{\text {max }} \\ A_{\text {max }} e^{j \theta(n)}, & \text { if }|x(n)|>A_{\text {max }}\end{cases}
$$

where $\theta(n)=\arg [x(n)]$. Therefore the magnitude of the clipped signal does not exceed the threshold $\mathrm{A}_{\max }$ and the phase of $\mathrm{x}(\mathrm{n})$ is preserved. The clipping severity is measured by the clipping ratio and defined as:

$$
\mathrm{CR}=\frac{\mathrm{A}_{\max }}{\sqrt{\mathrm{P}_{\mathrm{s}}}}
$$

where $P_{s}$ is the average power of $x(n)$ and it is given by:

$$
\mathrm{P}_{\mathrm{s}}=\frac{1}{\mathrm{~N}} \sum_{\mathrm{n}=0}^{\mathrm{N}-1}|\mathrm{x}(\mathrm{n})|^{2}
$$

The clipping causes both in-band and out-of-band distortion because of nonlinear operation of the clipping, the in-band distortion causes degradation of BER, while the distortion also caused out-of-band emission.

\section{The proposed hybrid method}

By combing the concepts of both the threshold and clipping techniques, a new formula can be proposed to reduce the PAPR of CWPM signal as follows:

$$
y(n)= \begin{cases}0 & \text { if }|x(n)|<T \\ x(n) & \text { if } T \leq|x(n)| \leq A_{\max } \\ A_{\max } e^{j \theta(n)} & \text { if } T \leq|x(n)|>A_{\max }\end{cases}
$$

The above formula is simply a composition of (4) and (7). The evaluation of the performance of the proposed method as compared with traditional ones for the same values of $\mathrm{T}$ and $\mathrm{A}_{\max }$ will considered in the next section.

\section{Simulation RESUlts}

A simulation model has been implemented for the CWPM system shown in Fig.1. The performance results of the PAPR reduction methods mentioned in section III are compared over frequency selective Rayleigh fading channel. The simulation parameters can be summarized as follows: the modulation type is DQPSK, the number of subcarriers is 128 , the wavelet family is Daubechies $6(\mathrm{db} 6)$ and the number of paths of the fading channel is two. The delay of the second path is 8Ts and the associated gain is $-8 \mathrm{~dB}$ while the Doppler spread factor $\mathrm{F}_{\mathrm{d}} \mathrm{T}_{\mathrm{s}}$ is 0.015 .

Fig. 2 shows the CCDF of the mean PAPR for the threshold method of different values of $\mathrm{T}$. It can be seen in this figure that the use of Donoho threshold (the threshold with minimum degradation in BER) achieves reduction in PAPR of about 2.5 $\mathrm{dB}$ at CCDF equals 0.01 . Fig. 3 shows the CCDF of the mean PAPR for the clipping method with different values of CR. Although as CR decreases the mean PAPR decreases, the simulations show that the optimum value of $\mathrm{CR}$ is $4 \mathrm{~dB}$ where minimum degradation of error has been obtained. At $C R=4 \mathrm{~dB}$ the clipping method achieves about $4 \mathrm{~dB}$ less mean PAPR for $\mathrm{CCDF}=0.01$. Fig. 4 and Fig. 5 show the performance of the proposed hybrid technique (TC-method). Fig.4 shows the CCDF of the PAPR for the proposed method for various values of CR where Donoho threshold is used as the threshold value while Fig.5 shows BER performance of proposed method for various values of CR with Donoho threshold too. From these two figures it can be seen that for $\mathrm{CR}=4 \mathrm{~dB}$, the proposed hybrid method achieves a reduction in mean PAPR 


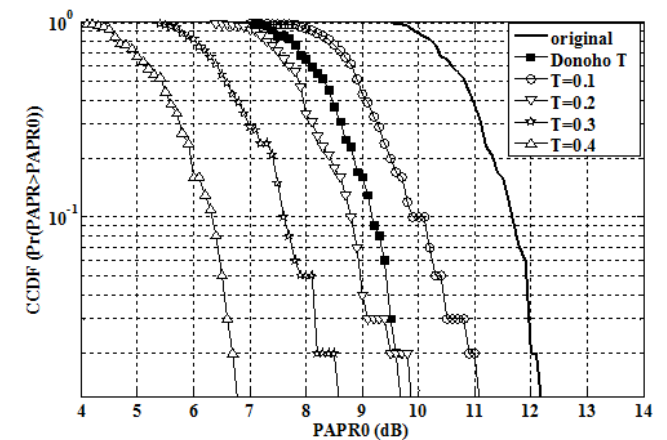

Figure 2. The CCDF of the PAPR of the threshold for various values of threshold.

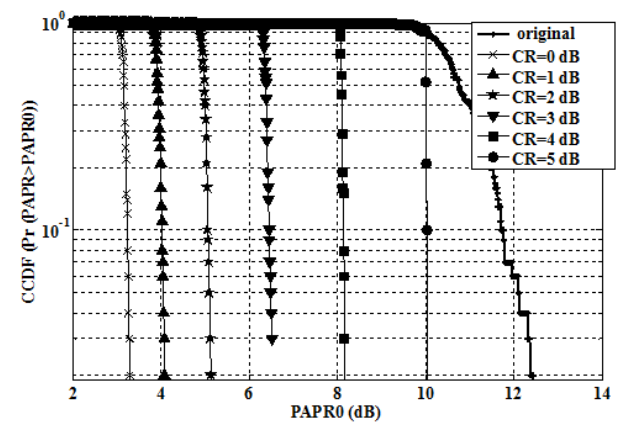

Figure 3. The CCDF of PAPR of clipping method for various value of clipping ratio.

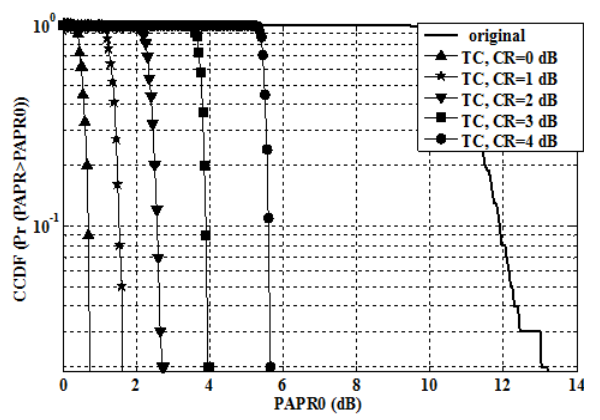

Figure 4. The CCDF of the PAPR of TC method with Donoho threshold for various values of clipping ratio.

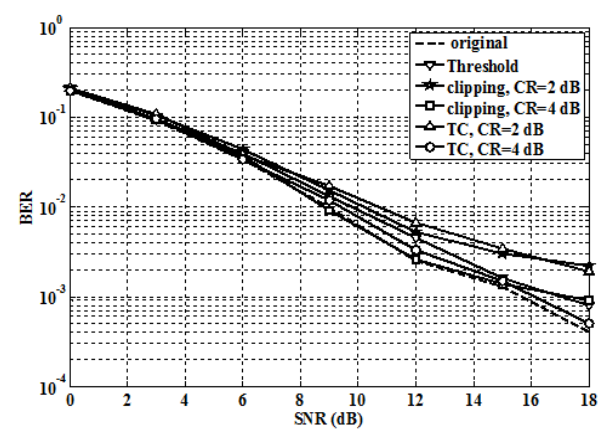

Figure 5. BER performance of CWPM that uses threshold, clipping and TC PAPR reduction methods. of about $7 \mathrm{~dB}$ at $\mathrm{CCDF}=0.01$ with $\mathrm{BER}$ degradation less than $0.5 \mathrm{~dB}$. This means that the TC method has achieved a reduction of $4.5 \mathrm{~dB}$ and $3 \mathrm{~dB}$ in PAPR over the threshold and clipping methods respectively for the same operation conditions.

\section{CONCLUSIONS}

In this paper the performance of three PAPR reduction techniques for CWPM has been evaluated. These are: threshold method, clipping method and a proposed technique that combines the preceding methods. The selection of the threshold value in the threshold method and the clipping ratio and in the clipping method is an optimization process. The optimum values are those whom provide the minimum mean PAPR and at the same time minimum degradation in BER. When the optimum threshold (Donoho) and clipping ratio $(4 \mathrm{~dB}$, for our simulation case) together in the proposed TC method a significant reduction in mean PAPR has been obtained with very small BER degradation as compared with threshold and clipping methods individually. Therefore the proposed TC method is good a candidate for the use to reduce PAPR in CWPM system. The possible drawback of the proposed scheme is its relative complexity since it requires the use of two successive PAPR reduction techniques.

\section{REFERENCES}

[1] A. Jamin and P. Mahonen, "Wavelet Packet Modulation for Wireless Communications," Wireless Communications and Mobile Computing Journal, Vol. 5, No. 2, pp. 123-137, Mar. 2005.

[2] M. Baro and J. Ilow, "Improved PAPR Reduction for Wavelet Packet Modulation using Multi-pass Tree Pruning," Proc. IEEE 18th Int. Symposium on Personal, Indoor and Mobile Radio Communications, September 2007.

[3] H. Zhang, D. Yuan, and M. Patzold, "Novel Study on PAPR Reduction in Wavelet Based Multicarrier Modulation System," Journal of Digital Signal Processing, Volume 17, No. 1, pp. 272-279, January 2007.

[4] N. T. Le, S. D. Muruganathan, and A. B. Sesay, "Peak-to-Average Power Ratio Reduction for Wavelet Packet Modulation Schemes via Basis Function Design," Proceedings of the IEEE Vehicular Technology Conference, September 2008.

[5] M. Rostamzadeh, V.T. Vakily, and M. Moshfegh, "PAPR Reduction in WPDM and OFDM System Using an Adaptive Threshold Companding Schemes," 5th IEEE International Multi-Conference on System, Signals and Devices SSD'08, 2008.

[6] B. Torun, M. K. Lakshmanan and H. Nikookar," Peak-to-Average Power Ratio Reduction of Wavelet Packet Modulation by Adaptive Phase Selection", 21st IEEE International. Symposium on Personal, Indoor and Mobile Radio Communications, September 2010.

[7] N. Kingsbury, "The Dual-Tree Complex Wavelet Transform: A New Technique for Shift Invariance and Directional Filters," in Proc. IEEE Digital Signal Processing Workshop, Bryce Canyon, UT, USA, No. 86, pp. 9-12, August 1998.

[8] H. N. Abdullah and F. S. Hasan, ""Performance Evaluation of Complex Wavelet Packet Modulation (CWPM) System over Multipath Rayleigh Fading Channel," Journal of signal and information processing, Vol.3, No.3, pp. 352-359, August 2012.

[9] D. L. Donoho, and I. M. Johnston, "Ideal Spatial Adaptation by Wavelet Shrinkage", Biometrika journal, Vol.81, No.3, pp.425-455, 1994.

[10] S. C. Thompson, J G. Proakis, and J. R. Zeidler," The Effectiveness of Signal Clipping for PAPR and Total Degradation Reduction in OFDM Systems", IEEE GLOBECOM proceedings, 2005. 